\title{
Effect of a submaxillary gland extract on Ehrlich tumor growth in mice
}

\section{P. W eill,
R. Frussa-Filho L.V. Bonamin ${ }^{1}$}

\author{
${ }^{1}$ Laboratório de Patologia, Instituto de Ciências da Saúde, \\ Universidade Paulista, São Paulo, SP, Brasil \\ 2Departamento de Farmacologia, Universidade Federal de São Paulo, \\ São Paulo, SP, Brasil
}

\section{Correspondence \\ L.V. Bonamin \\ Laboratório de Patologia, ICS \\ Universidade Paulista \\ Rua Dr. Bacelar, 1212 \\ 04026-002 São Paulo, SP \\ Brasil \\ Fax: +55-11-5012-1217 \\ E-mail: leoni@ sti.com.br \\ Publication supported by FAPESP.}

Received September 24, 1998 Accepted July 5, 1999

\section{Abstract}

Ablation of host submaxillary glands modifies Ehrlich tumor growth and tumor-infiltrating leukocytes, possibly by modifications in the serum level of growth factors produced by this gland. To extend this research, 7-month-old male EPM-1 mice $(\mathrm{N}=30)$ were divided into two groups: 1) inoculated with tumor cells previously incubated with submaxillary salivary gland extract (SGE) in PBS for $30 \mathrm{~min}$ at $37 \%$; 2 ) inoculated with tumor cells previously incubated with PBS, under the same conditions. Animals were inoculated into the footpad with 40 $\mu \mathrm{l}$ of a suspension containing $4.5 \times 10^{7}$ tumor cells $/ \mathrm{ml}$, and footpad thickness was measured daily for 10 days. Sections and smears of tumor cells were prepared from the tumor mass to determine mitosis frequency, percent of tumor cells immunopositive to nerve (NGF) and epidermal (EGF) growth factors and percent of tumor-infiltrating leukocytes. The incubation of tumor cells with SGE produced a tumor reduction of about $30 \%$ in size $(\mathrm{P}<0.01)$. This effect was not related to loss of cell viability during incubation, but a $33 \%$ increase $(\mathrm{P}<0.05)$ in the percentage of dead or dying tumor cells and a $15 \%$ increase in the percent of NGF/EGF-positive tumor cells $(\mathrm{P}<0.01)$ were observed in vivo at the end of experiment. Tumor-infiltrating lymphocytes and mitosis frequency did not differ between groups. These data suggest a direct effect of factors present in SGE on tumor cells, which induce degeneration of tumor cells.
The submaxillary salivary gland of mice and of other species has multiple functions in the body, including digestive, endocrine and immune functions (1). It produces and secretes several active peptides, some of which are growth factors, such as nerve growth factor (NGF), epidermal growth factor (EGF) (2) and $\alpha$-fetoprotein (3), which might be important for the growth of several tumors. Experimental studies on nude mice have

\section{Key words}

- Ehrlich ascites carcinoma - Submaxillary salivary gland - Growth factors shown that EGF administration and sialoadenectomy can modify human gastric tumor growth in nude mice (4).

We have demonstrated that the ablation of the submaxillary salivary glands in mice produces a significant decrease in Ehrlich tumor growth associated with an increase in NK cell population inside the tumor mass (5). The present study was undertaken to extend this research by using Ehrlich tumor 
cells pre-incubated with a submaxillary gland extract (SGE).

Seven-month-old male EPM-1 mice weighing 35 to $45 \mathrm{~g}$, housed under conditions of controlled temperature $\left(25 \pm 3^{\circ} \mathrm{C}\right)$ and light (12-h light/12-h dark, lights on at 6:00 a.m.), with food and water ad libitum, were used. The 7-month age of the animals was determined on the basis of previous data indicating that effects of growth factors are more evident in old animals (5).

The ascitic form of Ehrlich tumor was used to maintain tumor cells in our laboratory. For the experimental procedures, 3 to 4 $\mathrm{ml}$ of ascitic fluid from mice inoculated 12 days before were collected and centrifuged at $140 \mathrm{~g}$ for $10 \mathrm{~min}$. The supernatant was discarded and tumor cells were resuspended in phosphate-buffered saline (PBS), pH 7.2. The washing procedure was repeated 3 times.

The Trypan blue exclusion test was used to determine the percentage of living cells, which were counted in a modified Neubauer chamber. Cell viability was always at least $90 \%$, and the cell concentration for inoculation was $4.5 \times 10^{7}$ viable cells $/ \mathrm{ml}$.

SGE was prepared by the method of Skinner et al. (6). One hundred pairs of submaxillary glands were harvested from adult male mice and homogenized with a manual tissue homogenizer and $0.3 \mathrm{ml}$ of $0.2 \%(\mathrm{w} / \mathrm{v})$ soybean trypsin inhibitor (Sigma Chemical Co., St. Louis, MO, USA) and $7.7 \mathrm{ml} \mathrm{PBS} / \mathrm{g}$ gland were added at $4^{\circ} \mathrm{C}$. The homogenate was centrifuged at $20,000 \mathrm{~g}$ for $30 \mathrm{~min}$ at $4^{\circ} \mathrm{C}$ and the supernatant was stored at $-30^{\circ} \mathrm{C}$ until use.

Before inoculation, an aliquot of the cell suspension was incubated 1:1 with SGE for $30 \mathrm{~min}$ at $37^{\circ} \mathrm{C}$. The remaining cells were incubated with PBS for the same period of time and at the same temperature and proportion. Another Trypan blue exclusion test was carried out immediately after incubation to determine cell viability. A total of $0.04 \mathrm{ml}$ of these suspensions was inoculated into the hind footpad of mice, and paw thickness was measured daily for 10 days with a micrometer. The first measurement was made before tumor inoculation.

Mice were divided into two groups of 15 animals each: 1) mice inoculated with tumor cells previously incubated with SGE (group IE); 2) mice inoculated with tumor cells previously incubated with PBS (group IB).

After 11 days of tumor growth, animals were sacrificed and footpads removed. Footpads from half of the mice were fixed in $10 \%$ paraformaldehyde and processed histologically by usual embedding and staining methods (hematoxylin-eosin). These sections were used to count the number of mitoses per field, in five standard fields (40X) of each footpad section. A single cell suspension was obtained from the remaining footpads by mechanical dissociation of tumor cells in PBS. Several smears were prepared, fixed in $100 \%$ methanol at $4{ }^{\circ} \mathrm{C}$ for $10 \mathrm{~min}$ and stained by the immunocytochemical or Papanicolaou (PAP) method. All procedures were carried out using plastic material to avoid macrophage adhesion.

The Papanicolaou-stained smears were used to count the percentage of tumor cells and leukocytes and to differentiate viable cells from dead or dying tumor cells, macrophages and lymphocytes. Two hundred cells were counted in each smear. Degenerating cells were identified by volume reduction, cytoplasm irregularity, nuclear fragmentation and pyknosis.

The immunocytochemical procedure was carried out to identify NGF- and EGF-positive cells by the PAP method, using $0.1 \%$ periodic acid to block endogenous peroxidase activity and diaminobenzidine (Sigma) for developing, as described before (7). Nuclei were stained with Harry hematoxylin. A negative control was prepared with PBS replacing primary antibodies, used at 1:50 dilution and incubated at $37^{\circ} \mathrm{C}$ for $30 \mathrm{~min}$. A positive control was prepared with frozen sections of submaxillary salivary glands. Two hundred cells per smear were counted. 
Statistical analysis was performed using the Student $t$-test for tumor growth measurement (experimental $x$ control) and the Fisher test for cell counts. The level of significance was established at $\mathrm{P} \leq 0.05$.

There was a reduction of about $30 \%$ in tumor size when the tumor was previously incubated with SGE (Figure 1). There also was a $50 \%$ increase in the number of dead and dying cells in the group inoculated with SGE-pre-incubated tumor cells in relation to the control group, but the percentage of tumor-infiltrating macrophages and lymphocytes did not change. The percentages observed were $61 \%$ macrophages and $39 \%$ lymphocytes for the IE group and $49 \%$ macrophages and $51 \%$ of lymphocytes for the IB group. Cell viability did not change during incubation, as demonstrated by the Trypan blue test (data not shown).

No differences were observed in number of mitoses per field (data not shown), but the percent and the staining intensity of NGF/ EGF-positive cells were higher in tumors whose cells had been incubated with SGE before inoculation (IE group) in relation to the control (IB group) (Table 1 and Figure 2).

The effects of SGE on tumor growth seem to vary with type of treatment. The incubation of tumor cells with SGE a few minutes before inoculation into the footpad induced a marked reduction in tumor growth and an increase in the percentage of degenerated and NGF/EGF-positive cells compared to PBS-incubated group. These data suggest a direct interaction of one or more substances present in SGE with tumor cells during incubation, which could trigger intracellular mechanisms of cell destruction. This cell death would occur only after cell inoculation, because cell viability did not change immediately after incubation, excluding the possibility of cells being killed during manipulation. Thus, cell death occurred in vivo.

The reduction in tumor proliferation is not probable, because the number of mitoses did not change. The presence of tumor-infiltrating leukocytes was similar for the IE and IB groups, suggesting that tumor death was not determined by a cellular and/or inflammatory response to the tumor. However, it is known that NGF binding to neural cells induces apoptosis (8), and therefore a possible role of apoptosis mechanisms in the inhibition of Ehrlich tumor growth by SGE should be considered.

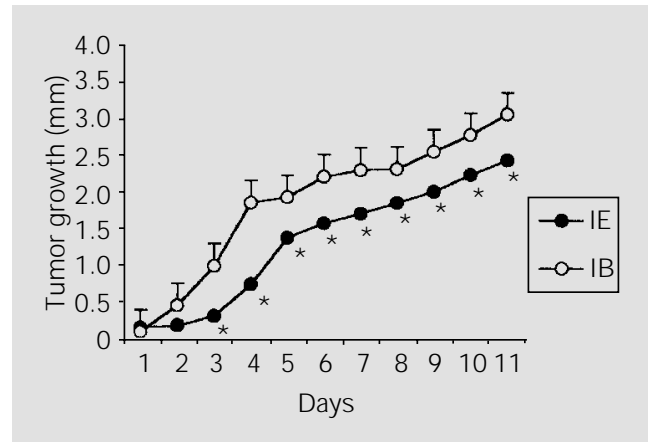

Figure 1 - Inhibition of tumor size by preincubation of Ehrlich cells with an extract of submandibular gland cells (SGE; IE group) or PBS (IB group). The experiment is described in the legend to Table 1. Data are reported as means \pm SD tumor size. $* \mathrm{P}<0.01$ compared to IB group (Student t-test).

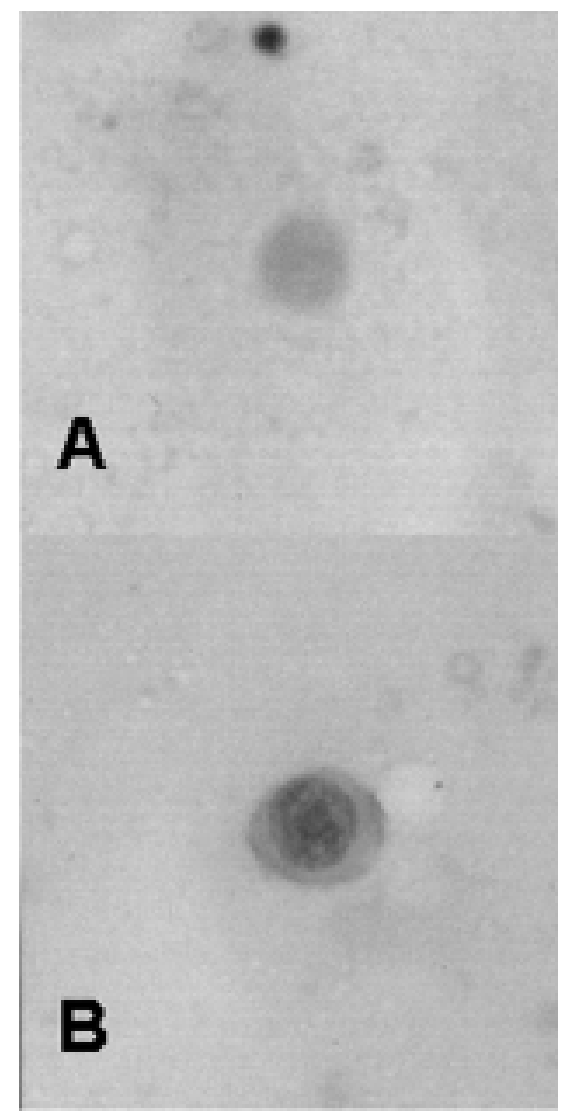

Figure 2 - Photomicrograph showing the NGF/EGF-positive tumor cells in groups IB (A) and IE (B). Note the differences in staining intensity between $A$ and B. 
Table 1 - Effect of pre-incubation of Ehrlich tumor cells on tumor viability and growth factor staining after inoculation into mouse foot pads.

The cells were incubated with an extract of salivary gland (IE) or PBS (IB) and inoculated into the foot pads of mice. Foot pad smears were obtained 11 days after inoculation and the distribution of tumor cells is based on counting 200 cells. Data are reported as means \pm SD for 15 animals in each group. NGF, Nerve growth factor; EGF, epidermal growth factor. $* \mathrm{P}<0.05$ compared to control group (Fisher test).

\begin{tabular}{lllcc} 
Groups & \multicolumn{3}{c}{ Tumor cells (\%) } \\
\cline { 2 - 5 } & Dead & Live & NGF + tumor & EGF + tumor \\
\hline IE & $45^{*}$ & 55 & $16^{*}$ & $12^{*}$ \\
IB & 12 & 88 & 7 & 1
\end{tabular}

These results appear to contradict those reported in previous studies, in which ablation of the submaxillary gland increased tumor infiltrating NK cells and reduced tumor growth (5), but the relation between biologically active factors from the submaxillary gland and tumor growth is highly complex and could be related either to cell degeneration or to cell differentiation and death (9). Thus, the response of tumor cells to withdrawal of endogenous factors by sialoadenectomy is not necessarily the opposite of their response to overexposure to the same submaxillary gland factors. On the other hand, direct cell treatment and overexposure to SGE decreased Ehrlich tumor development in vivo.

The inhibitory activity of excessive stimuli on biologic systems has been described by several investigators and was defined in the past as hormesis or Arndt-Schulz law: "A stimulus on a living cell elicits an activ- ity, which is inversely proportional to the intensity of the stimulus". This law was forgotten and even refuted by early scientists, but today it is known that some biological phenomena can present this behavior, such as corticoids in relation to macrophage activity and lymphocyte anergy after overexposure to antigens (10).

The substances that would lead to cell death in the present case are unknown, but the marked increase in staining and percentage of NGF/EGF-positive tumor cells incubated with SGE suggests that these growth factors themselves could be related to tumor cell death. In fact, this view is supported by the intra-nuclear immunohistochemical staining for NGF in Ehrlich tumor cells observed in previous studies (11). Tumor regression with NGF super-stimulation has been previously reported in human prolactinomas (12). In addition, tumor growth inhibition by NGF was observed also in rat $\mathrm{T} 9$ anaplastic glioma cells, in a dose-dependent manner, and was attributable to a gradual accumulation of growth-arrested cells at the G1 phase (13).

Although in the present study the specific role of NGF/EGF in tumor growth changes was not determined, the unexpected inhibitory response of tumor cells when incubated with SGE could indicate a non-linear relationship between submaxillary salivary gland factors and tumor growth, whatever the factor(s) involved. This finding raises the question about the possibility of the same response being obtained with apparently opposite stimuli (ablation of the gland or overexposure during incubation with the gland extract) and the necessity to understand this pattern of biological facts.

\section{References}

1. Mathison R, Davison J $\mathrm{S} \&$ Befus $A D$ (1994). Neuroendocrine regulation in inflammation and tissue repair by submandibular gland factors. Immunology Today, 15: 527-532.
2. Barka T (1980). Biologically active polypeptides in submandibular glands. J ournal of Histochemistry and Cytochemistry, 28: 836-859.

3. Tsuji T \& Nagai N (1993). Production of alpha-fetoprotein by human submandibular gland. International J ournal of Developmental Biology, 37: 497-498.

4. Okuda T, Onda M, Tokunaga A \& Sugisak $Y$ (1994). Stimulatory effect of EGF and 
inhibitory effect of sialoadenectomy on growth of an EGF receptor-hyperproducing human gastric cancer xenograft in nude mice. Surgery Today, 24: 725-733.

5. Bonamin LV (1995). The role of NGF in the Ehrlich tumor's biology. Doctoral thesis, FMVZ, USP, São Paulo.

6. Skinner KA, Soper BD \& Tepperman BL (1984). Effect of sialoadenectomy and salivary gland extracts on gastrointestinal mucosal growth and gastrin levels in the rat. J ournal of Physiology, 351: 1-12.

7. Bonamin LV \& Malucelli BE (1996). Substance $\mathrm{P}$ does not modify mononuclear cell innervation on Ehrlich tumor growth in mice. Brazilian J ournal of Medical and Biological Research, 29: 359-362.

8. Rabizadeh S, Oh J, Zhong LT, Yang J,
Bitter CM, Butcher LL \& Bredesen DE (1993). Induction of apoptosis by the lowaffinity NGF receptor. Science, 261: 345348.

9. Yaeger MJ, Koestner A, Marushige $K \&$ Marushige $Y$ (1991). The reverse transforming effects of nerve growth factor on five human neurogenic tumor cell lines: in vitro results. Acta Neuropathologica, 83: 72-80.

10. Oberbaum $M \&$ Cambar J (1994). Hormesis: Dose dependent reverse effects of low and very low doses. In: Endler PC \& Schulte J (Editors), Ultra High Dilution Physiology and Physics. Kluwer Academic Publishers, Dordrecht, 5-18.

11. Bonamin LV, Marchal T\& Malucelli BE (1994). Role of SP and NGF in Ehrlich tumor growth: an immunohistochemical study. Abstracts of the XII International Congress of Pharmacology. Canadian J ournal of Physiology and Pharmacology, 72 (Suppl 1): 257.

12. Missale $C$, Boroni $F$, Losa $M$, Giovanelli M, Zanellato A, Dal Toso R, Balsari A \& Spano $P$ (1993). Nerve growth factor suppresses the transforming phenotype of human prolactinomas. Proceedings of the National Academy of Sciences, USA, 90: 7961-7965.

13. Marushige $Y$, Marushige $K \&$ Koestner $A$ (1992). Growth inhibition of anaplastic cells by nerve growth factor. Anticancer Research, 12: 2069-2073. 


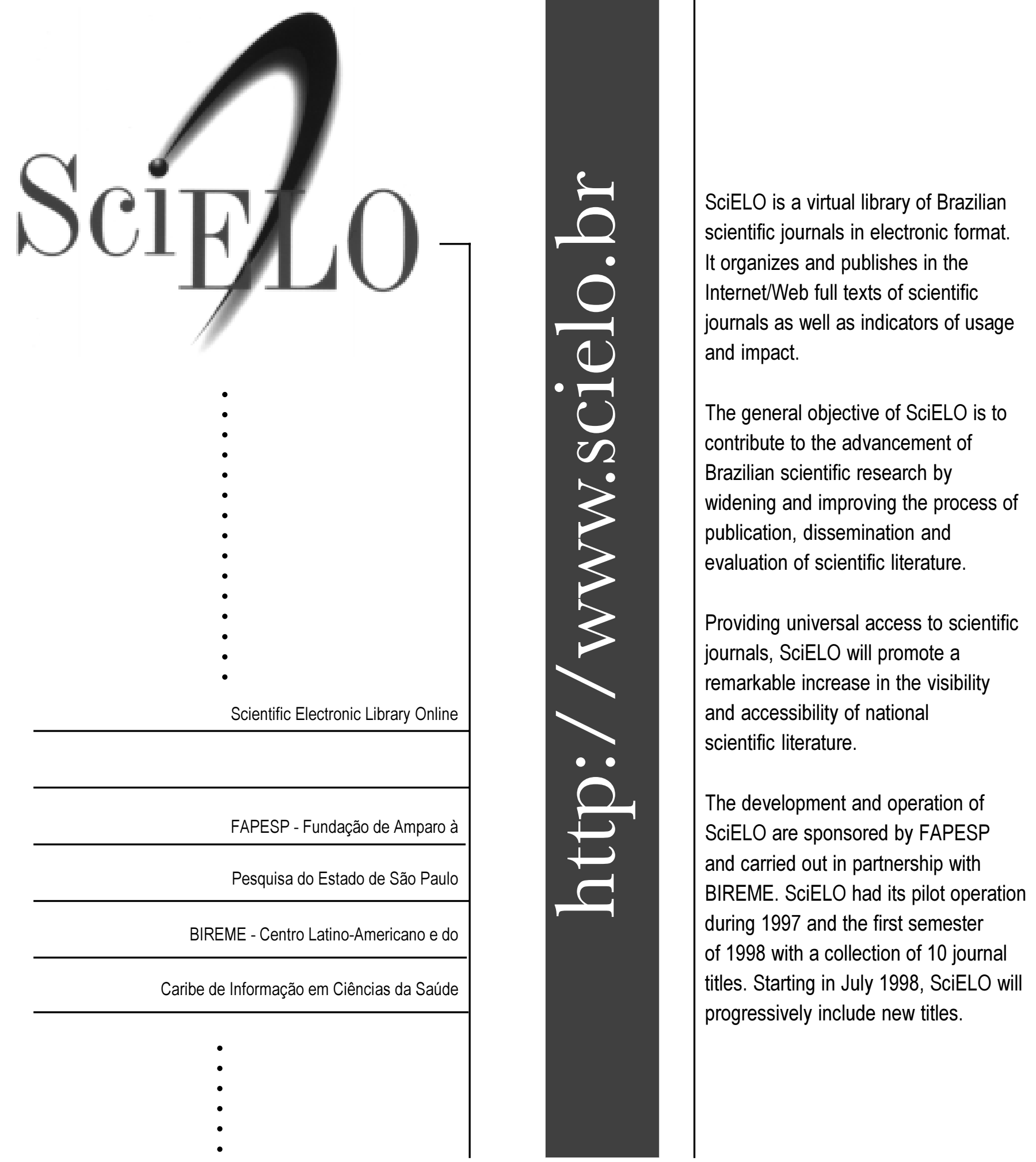

